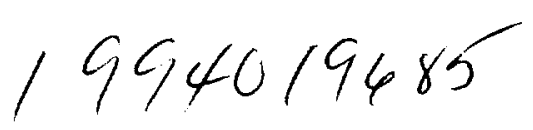

Center for Turbulence Research

Annual Research Briefs 1993

\title{
The production of premixed flame surface area in turbulent shear flow
}

\author{
By A. Trouvé
}

\section{Motivation and objectives}

In the flamelet theory, the modeling of the mean reaction rate is based primarily on a statistical description of the wrinkling of the flame surface due to the turbulent motions. The amount of wrinkling is quantified by the mean flame surface-tovolume ratio, also called the flame surface density. The flamelet theory produces an exact evolution equation for the flame surface density, called the $\Sigma$-equation, where basic physical mechanisms such as production by hydrodynamic straining and dissipation by propagation effects are described explicitly. In flamelet models, the flame surface density is obtained via a modeled formulation of this exact $\Sigma$-equation (Marble \& Broadwell 1977; Darabiha et al. 1987; Pope \& Cheng 1988; Maistret et al. 1989; Candel et al. 1990; Cant, Pope \& Bray 1990; Borghi 1990; Mantel \& Borghi 1991). Recent work at CTR, aimed at improving current models for the $\Sigma$-equation, focused primarily on the production and dissipation terms due to the turbulent flow field (Meneveau \& Poinsot 1991; Trouvé \& Poinsot 1993). In these studies, the turbulence upstream of the flame is considered isotropic and the mean flow velocity gradient tensor, $\left[\partial \widetilde{U}_{i} / \partial x_{j}\right]$, features only one non-zero component that corresponds to the mean flow acceleration through the combustion zone. Flame configurations featuring more complex mean deformation patterns, as found in practical systems where the combustion occurs in shear layers, boundary layers, or stagnation point Hows, have not been considered yet.

There is, in fact, little knowledge on the influence of mean velocity gradients on the dynamics of premixed flame surfaces. In current flamelet models, since the turbulent flame stretch in the equation for $\Sigma$ depends on the turbulent kinetic energy, $k$, and its rate of dissipation, $\epsilon$, the effects of mean deformations are incorporated implicitly through their impact on $k$ and $\epsilon$. An additional term that is linearly related to $\left[\partial \widetilde{U}_{i} / \partial x_{j}\right]$ is also sometimes introduced to account for possible direct effects (see Duclos, Poinsot \& Veynante 1993).

In the present work, we use three-dimensional Direct Numerical Simulation (DNS) of prernixed flames in turbulent shear flow to characterize the effect of a mean shear motion on flame surface production. The shear is uniform in the unburnt gas, and simulations are performed for different values of the mean shear rate, $S$. The data base is then used to estimate and compare the different terms appearing in the $\Sigma$-equation as a function of $S$. The analysis gives in particular the relative weights of the turbulent flow and mean flow components, $\widehat{a_{T}}$ and $\widehat{A_{T}}$, of the flame surface production term. This comparison indicates whether the dominant effects of a mean flow velocity gradient on flame surface area are implicit and scale with the modified turbulent flow parameters, $k$ and $\epsilon$, or explicit and scale directly with the rate of deformation. 


\subsection{The evolution equation for the flame surface density}

In the flamelet regime, it is convenient to describe the flame-flow interactions in terms of the following two basic ingredients: a flame speed that characterizes the mean combustion intensity, and a surface area that characterizes the flame wrinkling (Williams 1985; Peters 1986; Bray 1990; Pope 1990; Poinsot, Veynante \& Candel 1991). For instance, the mean reaction rate may be written as a mean integrated chemical rate times the flame surface density:

$$
\left\langle\dot{\omega}_{R}\right\rangle=\left\langle\int \dot{\omega}_{R} d n\right\rangle_{S}\left\langle\Sigma^{\prime}\right\rangle=\left\langle\int \dot{\omega}_{R} d n\right\rangle_{S} \Sigma
$$

where $\dot{\omega}_{R}$ is the mass of fuel consumed per unit time and per unit volume; $\int \dot{\omega}_{R} d n$ is the local integral of the reaction rate along the flame normal direction; $\Sigma^{\prime}$ is the flame surface area per unit volume; $\Sigma$ is the flame surface density, defined as the expected value for $\Sigma^{\prime}, \Sigma \equiv\left\langle\Sigma^{\prime}\right\rangle$. Note that in (1), all quantities are local and mean operators correspond to ensemble-averaging. While (\rangle denotes a standard, unweighted ensemble-average, \langle\rangle$_{S}$ denotes a surface mean, defined as an areaweighted ensemble-average (Pope 1988), $\langle Q\rangle_{S} \equiv\left\langle Q \Sigma^{\prime}\right\rangle /\left\langle\Sigma^{\prime}\right\rangle=\left\langle Q \Sigma^{\prime}\right\rangle / \Sigma$.

In (1), the integral of the reaction rate can be replaced by the local fuel consumption speed, $S_{C}$, defined as:

$$
S_{C} \equiv \frac{1}{\rho_{u} Y_{R, u}} \int \dot{\omega}_{R} d n
$$

where $\rho_{u}$ and $Y_{R, u}$ are respectively the density and the fuel mass fraction in the unburnt gas. The mean reaction rate may then be expressed as the surface mean of $S_{C}$, called the flamelet speed, times the flame surface density:

$$
\left\langle\dot{\omega}_{R}\right\rangle=\left(\rho_{u} Y_{R, u}\left\langle S_{C}\right\rangle_{S}\right) \Sigma .
$$

(3) is the classical flamelet expression for the ensemble mean of the reaction rate. In this expression, the flamelet speed, $\left\langle S_{C}\right\rangle_{S}$, accounts for local variations of the reaction rate along the flame surface. Recent direct simulations suggest that for flame Lewis numbers close to unity, the departures of $\left\langle S_{C}\right\rangle_{S}$ from the laminar consumption rate tend to average out when integrated across the whole turbulent flame (Ashurst, Peters \& Smooke 1987; Haworth \& Poinsot 1992; Rutland \& Trouvé 1993; Trouvé \& Poinsot 1993). In this situation, the flame surface density is to first order the single key quantity that determines the mean reaction rate. Note that Trouvé \& Poinsot (1993) also describe one flame with thermal-diffusive instability where the effect of the turbulence on the flame surface area is coupled with a significant increase in the mean flame intensity. In that case, both the flamelet speed and the flame surface density need to be modeled to determine the mean reaction rate.

In many situations, the principal effect of the turbulence is for the fluctuating velocity field to wrinkle the flame and greatly increase its surface area. This phenomenon is represented in ( 3 ) by the flame surface density, $\Sigma$. Following the pioneer 
work of Marble \& Broadwell (1997), an exact balance equation for the flame surface density was derived by Pope (1988) and Candel \& Poinsot (1990):

$$
\frac{\partial \Sigma}{\partial t}+\nabla \cdot\langle\dot{\mathbf{X}}\rangle_{S} \Sigma=\langle\kappa\rangle_{S} \Sigma
$$

where $\dot{\mathbf{X}}$ is the velocity of the flame surface, given by the sum of the fluid velocity and the flame propagation speed in the normal direction: $\dot{X}=\mathbf{u}+w \mathbf{n} ; \mathbf{n}$ is the unit vector normal to the flame surface; and $\kappa$ is the flame stretch. A useful expression for $\kappa$ is in terms of strain rate, flame curvature, and flame propagation speed (Candel \& Poinsot 1990):

$$
\kappa=a_{T}+2 w k_{m},
$$

where $a_{T}$ is the rate of strain acting in the flame tangent plane: $a_{T}=\nabla \cdot \mathbf{u}-\mathbf{n n}: \nabla \mathbf{u}$ (we use here standard tensorial notations: $\mathbf{n n}: \nabla \mathbf{u}=n_{i} n_{j} \partial u_{i} / \partial x_{j}$ ); and $k_{m}$ is the flame surface curvature, as given by the divergence of the flame normal direction: $2 k_{m}=\nabla . n$. In (5), positive curvature is chosen convex towards the reactants.

Equation (4) can be cast in various forms. For modeling purposes, it is useful to split the velocity vector into a mean component and a turbulent fluctuation: $\mathbf{u}=\widetilde{\mathbf{U}}+\mathbf{u}^{\prime \prime}$, where the tilde superscript denotes a Favre-averaged quantity: $\widetilde{\mathbf{U}}=$ $\langle\rho \mathbf{U}\rangle /\langle\rho\rangle$. We can then re-write (4) as follows:

$$
\frac{\partial \Sigma}{\partial t}+\nabla \cdot \tilde{\mathbf{U}} \Sigma+\nabla \cdot\left\langle\mathbf{u}^{\prime \prime}\right\rangle_{S} \Sigma+\nabla \cdot\langle w \mathbf{n}\rangle_{S} \Sigma=\left\langle A_{T}\right\rangle_{S} \Sigma+\left\langle a_{T}\right\rangle_{S} \Sigma+2\left\langle w k_{m}\right\rangle_{S} \Sigma,
$$

where we use the following notations:

$$
\begin{aligned}
& \left\langle A_{T}\right\rangle_{S}=\nabla \tilde{\mathbf{U}}-\langle\mathbf{n n}\rangle_{S}: \nabla \widetilde{\mathbf{U}} \\
& \left\langle\boldsymbol{a}_{T}\right\rangle_{S}=\left\langle\nabla \cdot \mathbf{u}^{\prime \prime}-\mathbf{n n}: \nabla \mathbf{u}^{\prime \prime}\right\rangle_{S} .
\end{aligned}
$$

The three convective terms on the left-hand side of $(6)$ are transport terms that correspond respectively to convection by the mean flow, turbulent diffusion, and flame propagation. The terms on the right-hand side of the equation are the source and sink terms for the flame surface density: $\left\langle A_{T}\right\rangle_{S}$ is the tangential strain rate due to the mean flow field; $\left\langle a_{T}\right\rangle_{S}$ is the strain rate due to the turbulent flow field; and $2\left\langle w k_{m}\right\rangle_{S}$ is a term that accounts for the combined effects of flame curvature and flame propagation.

We are particularly interested in this study in the effect of mean flow deformations on the dynamics of flame surface densities. As seen in the equation for $\Sigma$, in the presence of mean flow velocity gradients, $\partial \widetilde{U}_{i} / \partial x_{j}$, the flame surface is modified in two different ways: first by a rapid distortion that accounts for the direct straining of the flame surface by the mean velocity gradients (this effect is represented in (6) by $\left.\left(A_{T}\right\rangle_{S}\right)$ and second by a slower modification resulting from the adjustment of the turbulence to the applied deformations and from the corresponding variations in the turbulent flame stretch (this effect is represented mainly by $\left\langle a_{T}\right\rangle_{S}$ and $2\left\langle w k_{m}\right\rangle_{S}$ ). 


\subsection{Direct numerical simulation of turbulent premixed flames}

We use DNS to analyze the different terms in the equation for the flame surface density. The simulations are performed using a three-dimensional, compressible Navier-Stokes solver that fully resolves the turbulent flow field. The solver uses a high-order finite difference scheme: spatial derivatives are computed with a modified Padé scheme that is sixth-order accurate (Lele 1992); solutions are advanced in time with a third-order Runge-Kutta method (Wray 1990); boundary conditions are specified with the NSCBC method (Poinsot \& Lele 1992). We refer the reader to Lele (1992) and Poinsot \& Lele (1992) for further details concerning the system of equations solved and the numerical methods.

Because of the otherwise prohibitive computational cost, simulations are limited to simple but finite-rate reaction schemes. In this work, the chemistry model is a single step, irreversible chemical reaction where the reaction rate depends exponentially on temperature (Arrhenius kinetics):

$$
\dot{\omega}_{R}=B \rho Y_{R} \exp \left(-\frac{T_{a}}{T}\right),
$$

where $T_{a}$ is the activation temperature and $B$ is a constant that is determined according to the selected laminar flame speed. This formulation corresponds to a binary reaction in which one of the reactants, $Y_{R}$, is strongly deficient, for example, in fuel-lean combustion. Also, it is worth emphasizing that the simulations do not use the constant density assumption; the reaction is exothermic and heat release effects are fully accounted for.

Following Williams (1985), we re-write the reaction rate as:

$$
\dot{\omega}_{R}=\Lambda \rho Y_{R} \exp \left(\frac{-\beta(1-\Theta)}{1-\alpha(1-\Theta)}\right)
$$

where $\Theta$ is the reduced temperature, $\Theta=\left(T-T_{u}\right) /\left(T_{b}-T_{u}\right) ; T_{u}$ is the temperature of the fresh reactants; $T_{b}$ is the adiabatic flame temperature; and the coefficients $\Lambda, \alpha$, and $\beta$ are, respectively, the reduced pre-exponential factor, the heat release factor, and the reduced activation energy:

$$
\Lambda=B \exp (-\beta / \alpha), \quad \alpha=\left(T_{b}-T_{u}\right) / T_{b}, \quad \text { and } \quad \beta=\alpha T_{a} / T_{b}
$$

The values of the flame parameters are reported in Table 1.

Another important feature of the simulations is that the transport coefficients are temperature dependent. These coefficients satisfy the following relations:

$$
\mu=\mu_{u}\left(T / T_{u}\right)^{b}, L e=\lambda_{t h} / \rho D c_{p}=\text { constant }, \operatorname{Pr}=\mu c_{p} / \lambda_{t h}=\text { constant },
$$

where $\mu, \lambda_{t h}$, and $D$ are the molecular diffusivities of, respectively, momentum, internal energy, and species mass; $c_{p}$ is the specific heat at constant pressure; $b$ is a constant; and $L e$ and $P r$ are respectively the Lewis number and the Prandt number. The simulations were performed for a non-unity Lewis number, $L e=0.8$. 


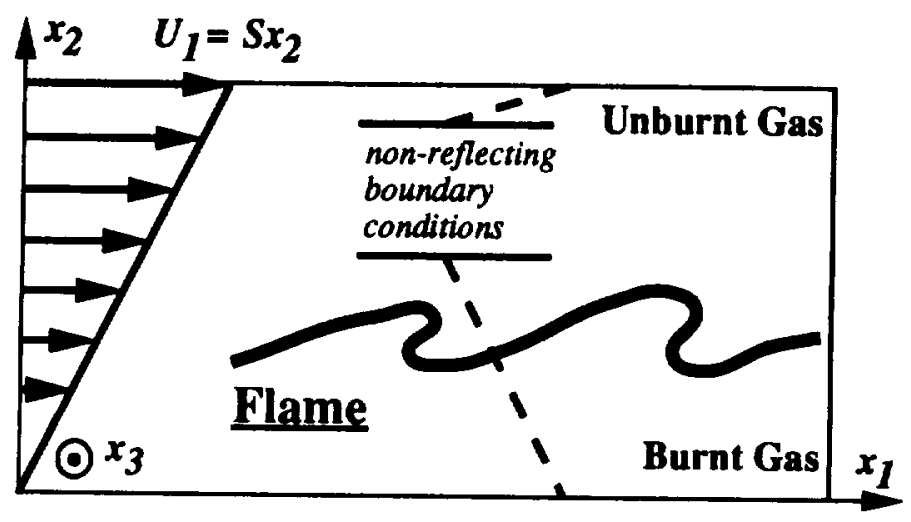

FIGURE 1. Numerical configuration.

\begin{tabular}{llllll}
\hline$L e$ & $\alpha$ & $\beta$ & $\Lambda^{+}$ & $b$ & $\operatorname{Pr}$ \\
\hline 0.8 & 0.75 & 8.0 & 456 & 0.76 & 0.75
\end{tabular}

TABLE 1. Dimensionless flame parameters, $\Lambda$ is made non-dimensional by the laminar flame time, $\Lambda^{+}=\Lambda\left(\lambda_{t h} / \rho c_{p}\right)_{u} / s_{L}{ }^{2}$.

The computational configuration corresponds to a premixed flame embedded in a three-dimensional, turbulent shear flow (see figure 1). The calculations are initialized with fresh reactants on the top side of the domain $\left(x_{2}>0\right)$ and burnt products on the bottom $\left(x_{2}<0\right)$; the two are separated by a plane laminar flame. The turbulence is initially isotropic, its velocity field being specified according to a model energy spectrum:

$$
E(k)=C\left(\frac{k}{k_{i}}\right)^{4} \exp \left\{-2\left(\frac{k}{k_{i}}\right)^{2}\right\},
$$

where $k$ is the wavenumber and $C$ and $k_{i}$ are model parameters that are specified according to, respectively, the initial turbulent rms velocity, $u^{\prime \prime}$, and integral length scale, $l$.

At $t=0$, a simple shearing motion, $\partial \widetilde{U}_{1} / \partial x_{2}=S$, is applied on the flow system. Note that due to the flow acceleration through the flame, the shear rate does not remain uniform in the cross-stream $x_{2}$ direction; in the flame zone, the velocity gradient $\partial \widetilde{U}_{1} / \partial x_{2}$ is a function of both time and cross-stream position. Outside the flame zone, however, the turbulence remains homogeneous in all three directions. In the burnt gas, the mean velocity gradient changes with time; it remains constant and equal to $S$ in the unburnt gas.

The top and bottom walls of the computational domain are inflow and outflow boundaries while periodic boundary conditions are applied in the $x_{1}$ and $x_{3}$ directions. Non-periodic boundary conditions are needed along $x_{2}$ for the following two 
reasons: to be able to maintain numerically a constant shear; to account for the density change from unburnt to burnt gases. Note, however, that no turbulence is generated at the inflow boundary, and the simulations are time-evolving rather than space-evolving.

Values of the run parameters are reported in Table 2. At time $t=0$, the turbulence is characterized by flow length scales that are a few times larger than the laminar flame thickness, $l / l_{F} \approx 4-5$ with $l_{F}=\left(\lambda_{t h} / \rho c_{p}\right)_{u} / s_{L}$, and turbulence intensities that are higher than the laminar flame speed, $u^{\prime \prime} / s_{L}>5$. The initial influence of the mean shear on the overall turbulence dynamics is measured by a time scale ratio, $S \tau_{0}$, where $\tau_{0}$ designates the initial, turbulent eddy turn-over time, $\tau_{0}=l / u^{\prime \prime}$. All simulated cases correspond to similar values of the initial Reynolds number. The initial turbulent Reynolds number based on the Taylor microscale, $\lambda$, is approximately $R e_{\lambda} \approx 25$. The initial turbulent Reynolds number based on the integral length scale is $R e_{l} \approx 35$. In Table 2 , a Damköhler number is also introduced using the Kolmogorov time scale and the laminar flame time: $D a=\left(\lambda / u^{\prime \prime}\right) /\left(l_{F} / s_{L}\right)=\sqrt{15}\left(\eta_{k} / v_{k}\right) /\left(l_{F} / s_{L}\right)$, where $\eta_{k}$ and $v_{k}$ are respectively the Kolmogorov length and velocity scales. While the simulated flames correspond to relatively low Damköhler numbers, $D a<1$, the reaction zone can still be described as a surface and the simulated flames occur in the flamelet regime.

Since in the presence of mean shear the turbulent structures are elongated in the streamwise direction, we use a computational domain that is twice as long in the streamwise direction compared to the cross-stream and spanwise directions. The grid spacing is uniform in all three directions; the grid resolution is $129 \times 129 \times 65$ and is, therefore, twice better in the direction of mean flame propagation, $x_{2}$. Also, given the growth of the turbulent length scales in the streamwise direction, the numerical simulations are all limited to relatively low total shears, $S t<15$, after which the eddies have sizes that are comparable to the computational domain and the numerical simulations suffer from insufficient resolution.

\begin{tabular}{lllllllll}
\hline Run designation & $S \tau_{0}$ & $R e_{l}$ & $R e_{\lambda}$ & $S l_{F} / s_{L}$ & $u^{\prime \prime} / s_{L}$ & $l / l_{F}$ & $D a$ & $L e$ \\
\hline Case 1 (lew08tSR) & 10.5 & 35. & 25. & 10. & 5.0 & 5.3 & 0.8 & 0.8 \\
Case 2 (lew08SR) & 7.0 & 35. & 25. & 6.7 & 5.0 & 5.3 & 0.8 & 0.8 \\
Case 3 (lew08sSR) & 3.5 & 35. & 25. & 3.3 & 5.0 & 5.3 & 0.8 & 0.8 \\
Case 4 (lew08wSR) & 3.3 & 38. & 28. & 6.7 & 7.5 & 3.8 & 0.4 & 0.8 \\
Case 5 (lew08uSR) & 1.7 & 38. & 28. & 3.3 & 7.5 & 3.8 & 0.4 & 0.8 \\
Case 6 (lew08vSR) & 0.7 & 38. & 28. & 1.3 & 7.5 & 3.8 & 0.4 & 0.8 \\
\hline
\end{tabular}

TABLE 2. Dimensionless parameters for the reacting flow simulations. All parameters correspond to the initial condition, $t=0$.

All terms in (6) can be obtained from the simulations. We refer the reader to Trouvé (1993) and Trouvé \& Poinsot (1993) for further details concerning the diagnostics that were developed to extract the relevant information from the DNS 


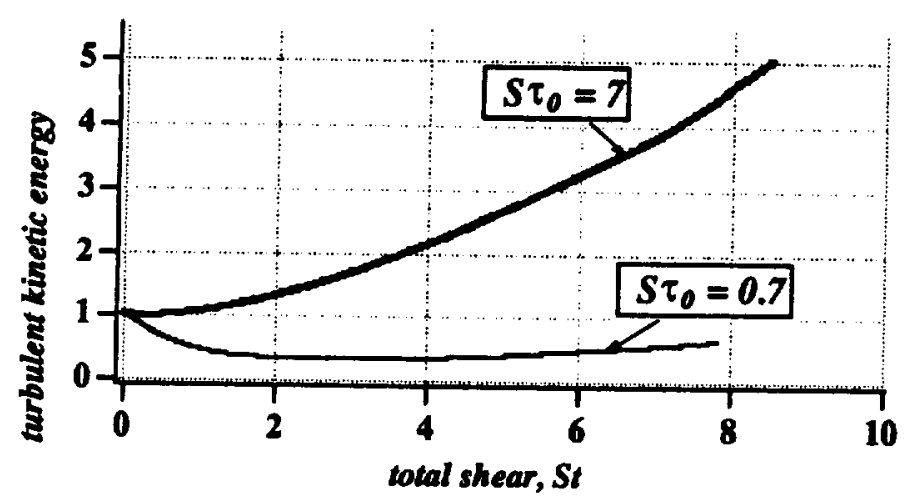

FIGURE 2. The effect of the turbulence-to-mean shear time scale ratio, $S \tau_{0}$, on the relative increase of total turbulent kinetic energy. A comparison between cases 2 and 6.

database. We simply briefly recall here that flame-based quantities are defined by making the assumption that the resolved finite-thickness reaction zone can be viewed as a constant progress variable surface, $c=c_{f}=0.8$ where $c \equiv 1-\left(Y_{R} / Y_{R, u}\right)$. Ensemble-averaging is conveniently performed in the homogeneous $x_{1}-x_{3}$ planes. For completion, we recall the expressions used to calculate the flame propagation speed, the flame surface density, and the surface means:

$$
\begin{gathered}
w=\dot{\mathbf{X}} \cdot \mathbf{n}-\mathbf{u} \cdot \mathbf{n}=\frac{1}{|\nabla c|} \frac{D c}{D t}=\frac{1}{\rho\left|\nabla Y_{R}\right|}\left(\dot{\omega}_{R}-\nabla\left(\rho D \nabla Y_{R}\right)\right), \\
\Sigma=\frac{1}{L_{x_{1}} L_{x_{3}}} \int_{c=c_{j}} \frac{|\nabla c|}{\sqrt{\left(\frac{\partial c}{\partial x_{1}}\right)^{2}+\left(\frac{\partial c}{\partial x_{3}}\right)^{2}}} d l,
\end{gathered}
$$

where $L_{x_{1}}$ and $L_{x_{3}}$ are the $x_{1}$ and $x_{3}$ dimensions of the computational domain.

$$
\langle Q\rangle_{S} \equiv \frac{\left\langle Q \Sigma^{\prime}\right\rangle}{\left\langle\Sigma^{\prime}\right\rangle}=\frac{\int_{c=c_{f}}\left(Q|\nabla c| d l / \sqrt{\left(\frac{\partial c}{\partial x_{1}}\right)^{2}+\left(\frac{\partial c}{\partial x_{3}}\right)^{2}}\right)}{\int_{c=c_{f}}\left(|\nabla c| d l / \sqrt{\left(\frac{\partial c}{\partial x_{1}}\right)^{2}+\left(\frac{\partial c}{\partial x_{3}}\right)^{2}}\right)}
$$

\section{Accomplishments}

The simulations describe the wrinkling of the flame zone by the flow as well as the transition of the flow field from isotropic decaying to anisotopic sheared turbulence. The mean shear rate, $S$, is used as a control parameter to modify the coupling between the mean flow, the turbulence, and the flame. Since the wrinkling of the flame is mainly driven by the turbulent motions in the unburnt gas, we first describe in $\$ 2.1$ the mean shear-turbulence interactions that modify the turbulence upstream of the flame. We then turn in $\$ 2.2$ to the impact of these modifications on the evolution of flame surface densities with emphasis on the effect of $S$ on the flame surface production term. 


\subsection{The effect of mean shear in homogeneous turbulent shear flow}

Turbulent shear flows present several additional levels of complication compared to isotropic turbulence because of the anisotropy of the flow, the production of turbulent kinetic energy by the mean strain, the possible amplification of hydrodynamic (Kelvin-Helmholtz) instabilities, and the resulting presence of large scale coherent structures. The general complexity of sheared turbulence is significantly reduced in the case of homogeneous shear flows, where the mean shear is uniform throughout the flow. For instance, such basic flow configurations are free of large scale hydrodynamic instabilities. In addition, homogeneous turbulent flows allow much simplified and more powerful statistical descriptions. Due to this increase in simplicity, homogeneous shear flows have been extensively studied in the literature, experimentally (Tavoularis \& Corrsin 1981; Rohr et al. 1988; Tavoularis \& Karnik 1989), as well as numerically (Rogallo 1981; Rogers \& Moin 1987; Lee, Kim \& Moin 1990; Holt, Koseff \& Ferziger 1992).

We are particularly interested in this work in the effect of changing the mean shear rate, $S$, on the energy levels and the structure of the turbulence. To characterize the effect of $S$, we performed a preliminary series of six simulations without flame. The run parameters are identical to the ones given in Table 2 , except for the absence of chemical reaction. The grid resolution is $129 \times 65 \times 65$. The results are used in the subsequent simulations with flame to describe the dynamics of the turbulence in the unburnt gas. We present in this section the main results from these non-reacting flow simulations.

The evolution of initially isotropic turbulence in a mean flow of uniform simple shearing motion, $S=d \widetilde{U}_{1} / d x_{2}=$ constant, can be described in terms of the following three dimensionless parameters: the total shear, $S t$, that gives a nondimensional time; the time scale ratio $S \tau_{0}$; and a turbulent Reynolds number, $R e_{l}=u^{\prime \prime} l / \nu$, taken at $t=0 . S \tau_{0}$ is a measure of the initial importance of the applied shear. $R e_{l}$ determines the impact of viscous effects. Both parameters serve to describe the effect of initial conditions, and their influence will be negligible at large times.

The present simulations, however, are limited to total shears of approximately 10. At time $S t=10$, the turbulent eddies remains in non-equilibrium. For instance, the quantity $S k / \epsilon$ is still evolving. It is generally believed that uniformly sheared turbulence will ultimately achieve a self-preserving state in which the turbulence changes at a rate independent of the initial conditions. In the simulations, the turbulence is clearly still far from this equilibrium state.

For small values of the total shear $S t$, the simulations reveal a range of flow regimes ranging from viscous decay for small values of $S \tau_{0}$, to rapid distortion for $S \tau_{0}>5$. This diversity in the turbulent flow response to the mean shear is shown in figure 2. While for $S \tau_{0}=0.7$ the flow goes through an initial phase characteristic of decaying grid turbulence and energy growth is only observed at later times, $S \tau_{0}>3$, for $S \tau_{0}=7$, the flow is dominated by the mean shear-turbulence interactions and the energy growth starts immediately at $t=0$.

Figures 3 and 4 describe the partition of turbulent kinetic energy between the 


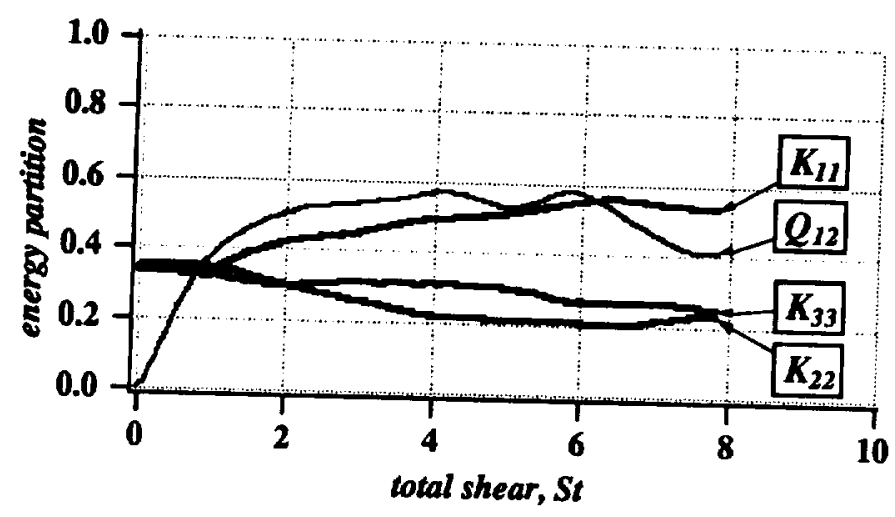
FIGURE $3(\mathrm{~A}) . \quad$ Partition of the turbulent kinetic energy, $k ; K_{i j} \equiv \widetilde{u_{i}^{\prime \prime} u_{j}^{\prime \prime} / 2 k}$
$Q_{12} \equiv K_{12} / \sqrt{K_{11}^{\prime} K_{22}}$. Case $6, S \tau_{0}=0.7$.

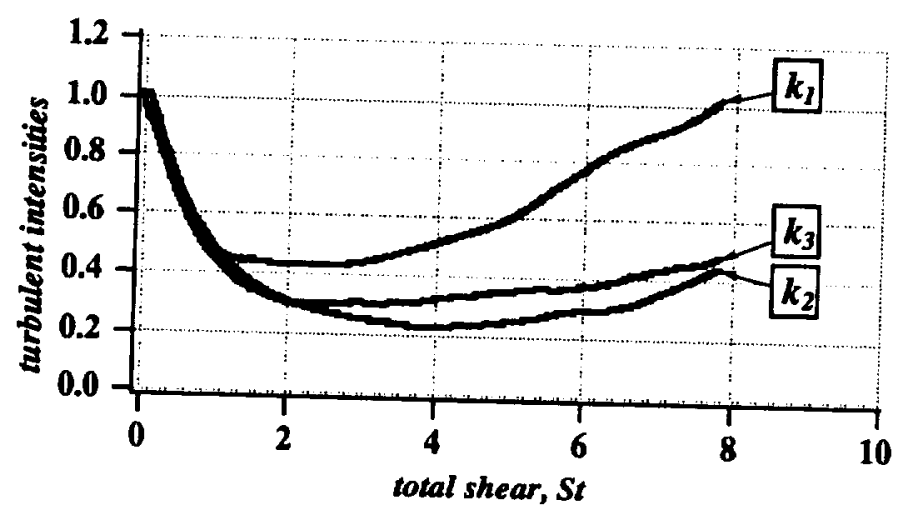

FIgURE 3(B). Partition of the turbulent kinetic energy, $k ; k_{i} \equiv \widetilde{u_{i}^{\prime \prime} u_{i}^{\prime \prime} / 2}=K_{i i} \times k$
Case $6, S \tau_{0}=0.7$.

streamwise, cross-stream, and spanwise velocity fluctuations. As seen in figures $3(a)$ and 4(a), the simulations feature an initial transient phase, $0 \leq S t \leq 6$, where the anisotropy levels, $K_{i j} \equiv \widetilde{u_{i}^{\prime \prime} u_{j}^{\prime \prime}} / 2 k$, change rapidly, followed by a phase of slower adjustment where the flow approaches structural equilibrium with $K_{i j}$ asymptoting to constant values. Note that the flow remains more isotropic in case 6 compared to case 2. This result is consistent with the findings by Lee, Kim \& Moin (1990), who show that the structure of shear-driven homogeneous turbulence changes drastically when the mean shear rate, $S$, is increased and that the degree of anisotropy of the flow increases with $S$.

Figures 3(b) and 4(b) compare the magnitudes of the different turbulent kinetic

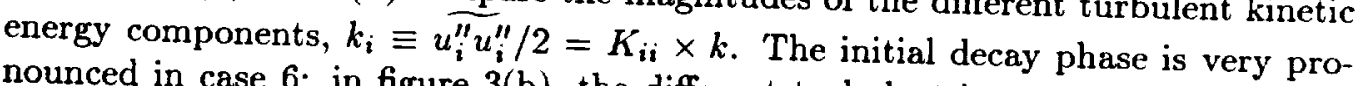
nounced in case $6:$ in figure $3(\mathrm{~b})$, the different turbulent intensities remain lower than their initial value up to $S t=8$. The situation is drastically different at high 


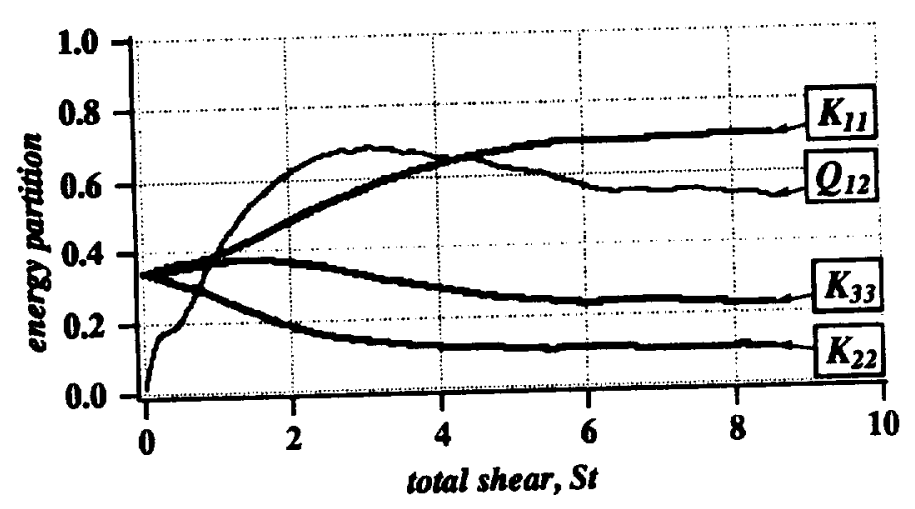

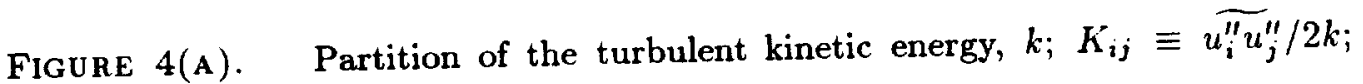
$Q_{12} \equiv K_{12} / \sqrt{K_{11} K_{22}}$. Case $2, S \tau_{0}=7$.

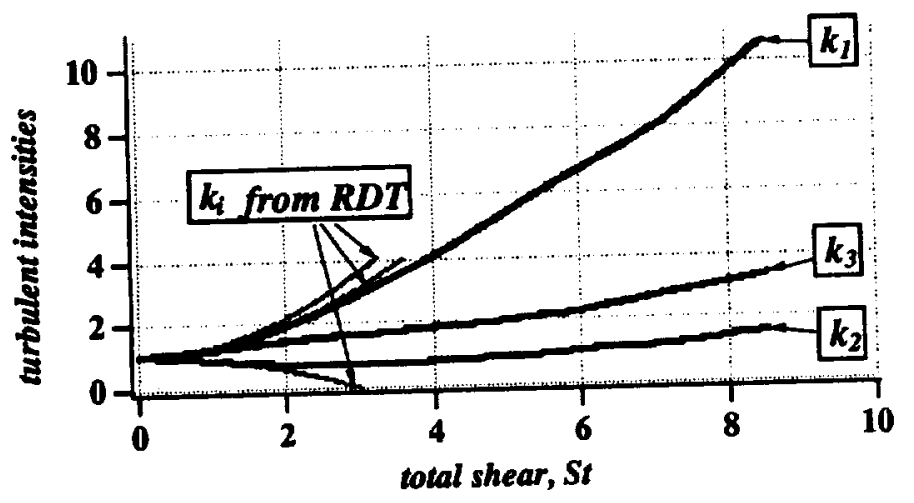

FIGURE 4(B). Partition of the turbulent kinetic energy, $k ; k_{i} \equiv \widetilde{u_{i}^{\prime \prime} u_{i}^{\prime \prime}} / 2=K_{i i} \times k$. Case 2, $S \tau_{0}=7$.

shear rates: in figure $4(\mathrm{~b})$, at $S t=8$, the streamwise component $k_{1}$ is already an order of magnitude larger than its initial value. Furthermore, the simulations feature the following classical arrangement: $k_{1}>k_{3}>k_{2}$. The first inequality reflects the fact that the streamwise component receives energy directly from the mean shear, while the other components are maintained by redistribution of energy through the pressure-strain correlation.

We also present in figure 4(b) a comparison between the DNS data and the predictions from Rapid Distortion Theory (RDT) (see for example Townsend 1976; Maxey 1982). RDT is a linear theory that applies to situations in which the mean shear-turbulence interactions dominate the nonlinear turbulence-turbulence interactions. Its domain of validity corresponds to $S \tau_{0} \gg 1$. RDT sheds light on the mechanisms of energy exchange between the different turbulent velocity components. For instance, RDT shows that while most of the energy is concentrated in the streamwise component, $k_{1}$, energy is also transferred from both $k_{1}$ and $k_{2}$ to $k_{3}$ 
by the pressure-strain correlation, which accounts for the net reduction (increase) in $k_{2}\left(k_{3}\right)$ by the shear distortion seen in figure $4(\mathrm{~b})$.

Hence, while the initial time evolutions of $k_{1}$ and $k_{3}$ depend strongly on the parameter $S \tau_{0}$, the cross-stream turbulent velocities are less sensitive to its value: for $S \tau_{0}<1, k_{2}$ decreases at small times because of viscous decay; for $S \tau_{0} \gg 1, k_{2}$ also decreases because of a net energy loss to $k_{3}$.

\subsection{The evolution of flame surface area in sheared turbulence}

It is well known that in the presence of mean shear, the turbulent rates of mixing and chemical reaction tend to be increased. One reason for this increase is the development of large scale coherent structures that are amplified by the Kelvin-Helmholtz hydrodynamic instability. This pseudo-laminar mechanism has been extensively studied in the last twenty years, including in premixed and nonpremixed combustion systems. In homogeneous turbulent shear layers, the hydrodynamic instability does not occur, and the enhancement of mixing and chemical reaction results from increased straining and increased turbulent transport due to shear-production of turbulent kinetic energy. This turbulent mechanism has been studied in a number of recent numerical studies in the context of mixing problems (Rogers, Mansour \& Reynolds 1989; Holt, Koseff \& Ferziger 1992) and nonpremixed chemical reactions (Leonard \& Hill 1992; Nomura \& Elghobashi 1992). Nomura \& Elghobashi assume in their study an infinitely fast reaction, while Leonard \& Hill consider a finite-rate reaction both with temperature-independent and temperature-dependent kinetics. Both studies are limited by the constant density assumption. In these studies, the enhancement of chemical reaction due to the mean shear is explained by the increased turbulent strain rates and increased reactant concentration gradients found along the flame surface. Since in the absence of quenching, higher concentration gradients are equivalent to higher reaction rates, it is suggested that one basic effect of mean shear is to increase the mean flame intensity. This effect is related in (3) to modifications of the flamelet speed, $\left\langle S_{C}\right\rangle_{S}$, not to modifications of the flame surface density, $\Sigma$.

In premixed flames, however, the departures of $\left\langle S_{C}\right\rangle_{S}$ from the laminar consumption rate tend to average out when integrated across the whole turbulent flame, and in many situations the mean combustion intensity is only weakly sensitive to the turbulence. Therefore, the argument that relates the increased combustion efficiency observed in nonpremixed flames with shear to a change in the flame structure is not expected to apply to premixed configurations. In that case, the effect of mean shear must be related to a modification of the total flame surface area. In the following, we use DNS to get basic information on the dynamics of flame surface densities as a function of the mean shear rate, $S$. The mean shear rate, $S$, is varied in the proportion of 1 to 10 , as seen in Table 2. Large values of the shear rate (as in cases 1,2 , and 4) are relevant to a flame propagating in the near-wall region of a turbulent channel flow. Lower values (as in case 6) can be found in flames stabilized in subsonic shear layers. 


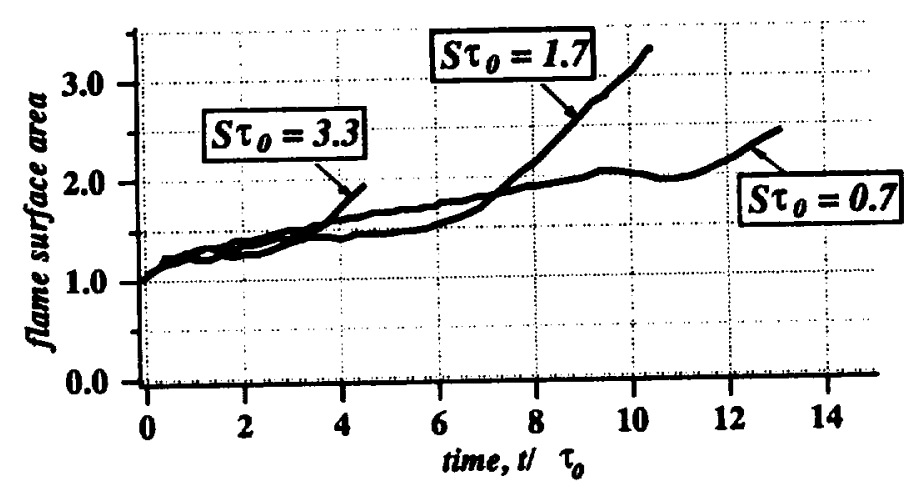

FiguRE 5(A). The effect of the turbulence-to-mean shear time scale ratio, $S \tau_{0}$, on the relative increase of total flame surface area, $\left\langle S_{V}\right\rangle / L_{x_{1}} L_{x_{3}}$. A comparison between cases 4,5 , and 6 . Time is made non-dimensional by the initial, turbulent eddy turn-over time, $\tau_{0}$.

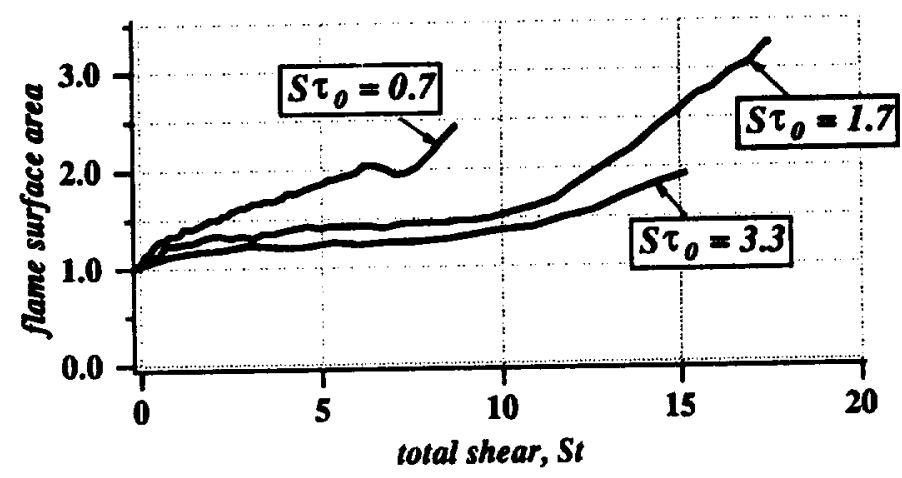

FigURE 5(B). The effect of the turbulence-to-mean shear time scale ratio, $S \tau_{0}$, on the relative increase of total flame surface area, $\left\langle S_{V}\right\rangle / L_{x_{1}} L_{x_{3}}$. A comparison between cases 4,5 , and 6 . Time is made non-dimensional by the mean shear rate, $S$.

\subsubsection{The total flame surface area}

As described in $§ 1.2$, six different cases have been simulated that correspond to turbulent flames characterized by the same laminar flame thickness, $l_{F}$, the same laminar flame speed, $s_{L}$, embedded in two different initial turbulent flow fields (in this respect cases 4,5 , and 6 differ from cases 1,2 , and 3 ), and with different values of the mean shear rate, $S$.

The effect of changing the shear rate on the production of flame surface area, $\left\langle S_{V}\right\rangle$, is shown in figure 5 for cases 4,5 , and 6 . In figure $5(\mathrm{a}),\left\langle S_{V}\right\rangle$ is plotted versus time, with time made non dimensional by $\tau_{0}$. Cases 4 and 5 feature an initial phase of slow growth followed by a phase of more rapid growth. In case 6 , the transition to this second phase is not observed. These two successive phases are 
more clearly seen when $\left\langle S_{V}\right\rangle$ is plotted as a function of total shear, $S t$ (figure $5(\mathrm{~b})$ ). For $0 \leq S t \leq 10$, the production of flame surface area is slow and the differences between all simulated cases remain moderate. In contrast, for $S t>10$, the flame surface is rapidly growing at a rate that does not seem to depend on $S$. In case 6 , the simulation is stopped at $S t \approx 8.5$, before the transition to the second phase is completed.

One important result in figure $5(\mathrm{~b})$ is that the flame response to the applied shear scales like the turbulent flow field (\$2.1) with the total shear, $S t$.

\subsubsection{The production and dissipation of flame surface area}

Using the diagnostics presented in $§ 1.2$, we now turn to the analysis of the source and sink terms of flame surface area as they appear on the right-hand-side of equation (6). These terms can be resolved spatially across the turbulent flame (Trouvé 1993; Trouvé \& Poinsot 1993). In the following, they are space-averaged in the cross-stream direction, and we limit our discussion to time variations of global flame properties. We estimate the global value of any quantity $Q$ using the following $\Sigma$-weighted space-averaging scheme:

$$
\widehat{Q}(t) \equiv\left(\int\langle Q\rangle_{S}\left(x_{2}, t\right) \Sigma\left(x_{2}, t\right) d x_{2}\right) /\left(\int \Sigma\left(x_{2}, t\right) d x_{2}\right) .
$$

Using (17), we define the total production of flame surface by hydrodynamic staining, $\widehat{P}$, the total dissipation by flame propagation effects, $\widehat{D}$, and the resulting net total flame stretch, $\widehat{\kappa}$ as follows:

$$
\begin{gathered}
\widehat{P}(t) \equiv \widehat{A_{T}}+\widehat{a_{T}}, \\
\widehat{D}(t) \equiv \widehat{2 w k_{m}}, \\
\widehat{\kappa}(t) \equiv \widehat{P}+\widehat{D} .
\end{gathered}
$$

Also, the total flame surface area, $\left\langle S_{V}\right\rangle$, is equal to the volume-integral of the flame surface density:

$$
\frac{\left\langle S_{V}\right\rangle(t)}{L_{x_{1}} L_{x_{3}}}=\int \Sigma\left(x_{2}, t\right) d x_{2}
$$

and, using (4), $\widehat{\kappa}$ can be directly related to the instantaneous rate of change of the total flame surface area in the computational domain:

$$
\widehat{\kappa}(t)=\frac{d\left\langle S_{V}\right\rangle}{d t} /\left\langle S_{V}\right\rangle
$$

Figure 6 shows $\widehat{P}, \hat{D}$, and $\widehat{\kappa}$ as a function of total shear for cases 4,5 , and 6 . It is seen that while the simulated cases exhibit large differences in the time history of the production term, $\widehat{P}$, these differences tend to be balanced by corresponding variations in the dissipation term, $\widehat{D}$, and the net total flame stretch, $\widehat{\kappa}$, follows approximately the same time evolution for all cases. In other words, the total 


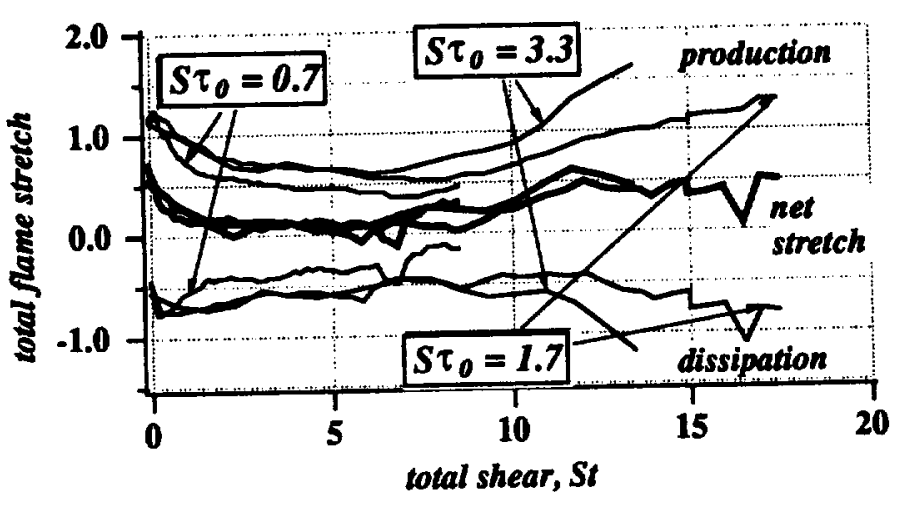

Figure 6. Time evolution of flame surface production, $\widehat{P}$, flame surface dissipation, $\widehat{D}$, and total flame stretch, $\widehat{\kappa}$. A comparison between cases 4,5 , and 6 . $\widehat{P}, \widehat{D}$, $\widehat{\kappa}$ are made non-dimensional by the laminar flame time, $\left(\lambda_{t h} / \rho c_{p}\right)_{u} / s_{L}{ }^{2}$.

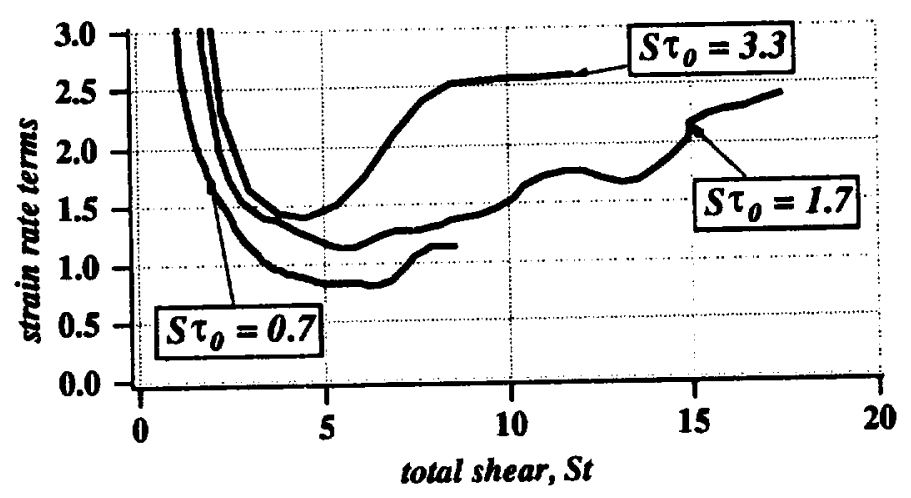

FIgURE 7. $\widehat{a_{T}} / \widehat{A_{T}}$ vs St. Relative weights of $\widehat{a_{T}}$ and $\widehat{A_{T}}$ in their contributions to flame surface production, $\widehat{P}$. A comparison between cases 4,5 , and 6 .

flame stretch scales with the total shear, $S t$, but does not depend on the parameter $S \tau_{0}$. It is worth emphasizing that this result is somewhat unexpected given the large impact of $S \tau_{0}$ on the turbulence dynamics as described in \$2.1. One quantity, however, that remains approximately the same from one simulation to the other is the cross-stream turbulent $\mathrm{rms}$ velocity, as measured by $k_{2}$. It is believed that the insensitivity of the total flame stretch to changes in $S \tau_{0}$ is a strong indication that $\hat{\kappa}$ scales with $k_{2}$ rather than $k$.

Furthermore, since $\widehat{\kappa}$ appears to be a function of $S t$ and not of $S \tau_{0},(22)$ can be re-written as:

$$
\left\langle S_{V}\right\rangle(S t)=\left\langle S_{V}\right\rangle(t=0) \exp \left(\frac{1}{S} \int_{0}^{S t} \widehat{\kappa}(T) d T\right)
$$

(23) shows that at constant total shear, $S t=$ constant, the flame surface area is a 


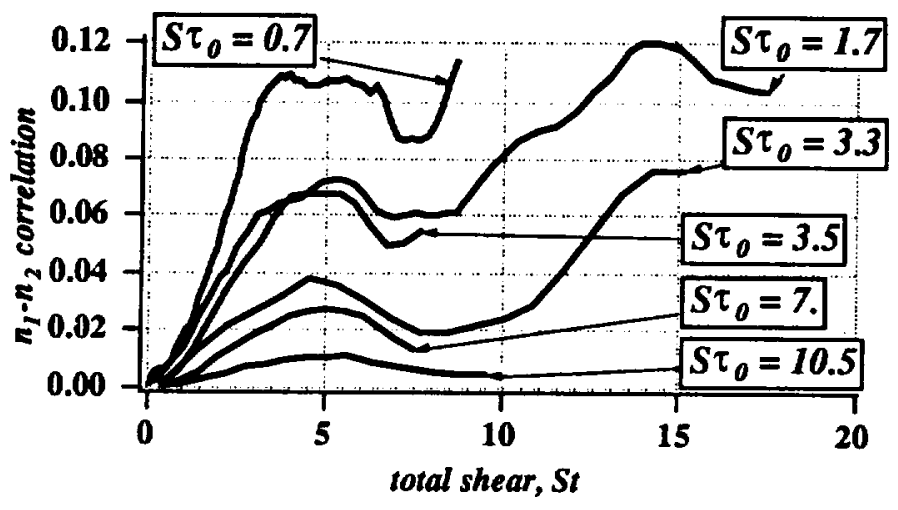

FIGURE $8(\mathrm{~A})$. The effect of $S$ on the magnitude of $-\widehat{n_{1} n_{2}}$. A comparison between cases 1 to 6 .

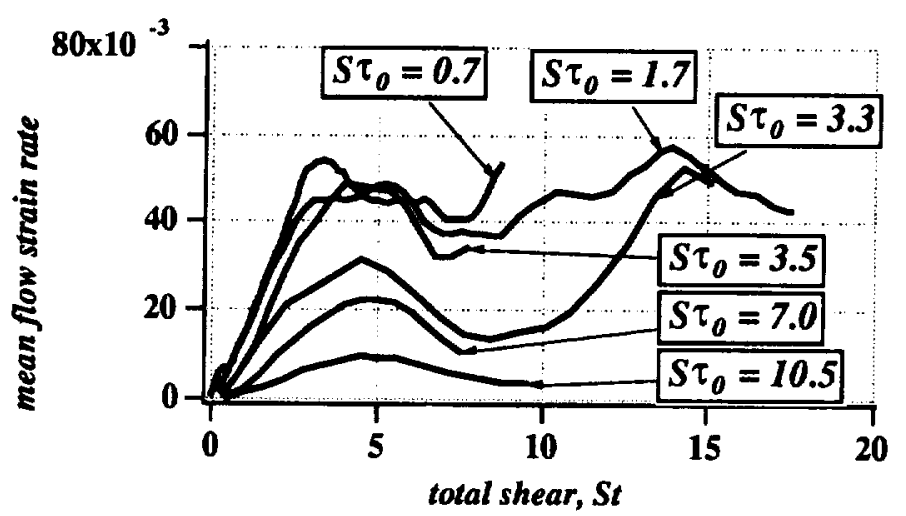

FIgURE $8(\mathrm{~B})$. The effect of $S$ on the magnitude of $\widehat{A_{T_{12}}}$. A cornparison between cases 1 to $6 . \widehat{A_{T_{12}}}$ is made non-dimensional by the mean shear rate $S$.

decreasing function of $S$, as was found in figure $5(\mathrm{~b})$.

As seen in (18), the mean tangential strain rate at the flame can be decomposed into two components: $\widehat{A_{T}}$ represents the contribution of the mean rate of deformation; and $\widehat{a_{T}}$ represents the effects of the turbulent flow velocity gradients. Figure 7 presents the ratio $\widehat{a_{T}} / \widehat{A_{T}}$ versus non-dimensional time for cases 4,5 , and 6 . At $t=0$, the flame is plane and there is no strain rate due to the mean flow field, $\widehat{A_{T}}=0$. As the flame gets wrinkled, the effect of the mean flow field gets more pronounced and $\widehat{A_{T}}$ and $\widehat{a_{T}}$ take comparable values. For $S t>5$, however, both the absolute value of $\widehat{a_{T}}$ and its relative weight in the expression for $\widehat{P}$ are increasing. These results indicate that a transition to a fully turbulent regime is occurring: for $S t>5$, the turbulence levels, $k_{1}, k_{3}$, and also $k_{2}$ are all increasing; the hydrodynamic straining at the flame, as measured by $\widehat{P}$, takes higher values; in the expression for $\widehat{P}, \widehat{a_{T}}$ is increasing faster than $\widehat{A_{T}}$ and the turbulent flow component of flame production is becoming dominant. 
We now take a closer look at the strain rate due to the mean flow field. $\left\langle A_{T}\right\rangle_{S}$ is a production term that depends on the mean cross-stream velocity gradient and the flamelets orientation:

$$
\left\langle A_{T}\right\rangle_{S}=\left(1-\left\langle n_{2}{ }^{2}\right\rangle_{S}\right) \frac{\partial \tilde{U}_{2}}{\partial x_{2}}-\left\langle n_{1} n_{2}\right\rangle_{S} \frac{\partial \tilde{U}_{1}}{\partial x_{2}}
$$

where $n_{i}$ is the $x_{i}$-component of the flame normal vector. The first term in (24) accounts for the flow acceleration through the flame; the second term describes the rapid distortion of the flame surface due to the applied shear. Both terms remain positive across the turbulent flame. Note that since in (24) the velocity gradients are multiplied by quantities that are direct measures of the turbulent flame wrinkling, the magnitude and relative weights of the two components of $\left\langle A_{T}\right\rangle_{S}$ are difficult to assess a priori. In the simulations, the statistics describing the flamelet's orientation do not vary significantly across the turbulent brush; $\left\langle n_{2}{ }^{2}\right\rangle_{S}$ and $\left\langle n_{1} n_{2}\right\rangle_{S}$ are functions of time but nearly independent of $x_{2}$ position. Therefore, space-averaging across the turbulent flame yields the following approximation:

$$
\widehat{A_{T}} \equiv \widehat{A_{T_{22}}}+\widehat{A_{T_{12}}} \approx \alpha \frac{\widehat{\partial \widetilde{U}_{2}}}{\partial x_{2}}+\beta \frac{\widehat{\partial \widetilde{U}_{1}}}{\partial x_{2}},
$$

where $\alpha$ and $\beta$ are constant coefficients.

Figure $8(\mathrm{a})$ presents the time variations of $-\widehat{n_{1} n_{2}}$ which can be considered as an estimate of $\beta$. All simulated cases follow the same time evolution when scaled in terms of $S t$ : growth from the initial zero value; saturation at $S t \approx 5$; short period of decrease; and rapid growth for $S t>8$. The magnitude of $-\widehat{n_{1} n_{2}}$ is a strong decreasing function of $S$. This result may be explained as follows. $-\widehat{n_{1} n_{2}}$ is determined by two simultaneous effects, a mean deformation effect and a turbulent diffusion effect. Let us consider, for instance, a sinusoidal flame surface of constant amplitude subjected at $t=0$ to a simple shearing motion: $-\widehat{n_{1} n_{2}}$ first increases, saturates at a time that scales with $S t$, and then decreases as the flame elements are turned away from the direction of maximum positive strain rate (inclined at 45 degrees from the mean flow direction) by the rotation component of the applied shear. In the simulations, this mean deformation effect is coupled with an increase of the turbulent flame thickness due to turbulent diffusion that is constantly counteracting the flattening of the flame due to the mean rotation. The characteristic time scale of the mean deformation effect is $1 / S$. The characteristic time scale of the turbulent diffusion effect is a turbulent time scale that is likely to scale with $1 / \sqrt{k_{2}}$. The largest magnitude for $-\widehat{n_{1} n_{2}}$ is obtained for cases where the turbulence dominates the mean deformation, that is for small values of $S$.

Hence, in (25) the increase of $\partial \widetilde{U_{1} / \partial} x_{2}$ with $S$ is cancelled by a corresponding decrease of the coefficient $\beta$. Figure $8(\mathrm{~b})$ illustrates this result and shows that the resulting strain rate is more than one order of magnitude smaller than $S$. Also, in all cases, $\alpha$ is always 5 to 10 times larger than $\beta$ and both terms in (25) have comparable contributions to $\widehat{A_{T}}$. 


\section{Conclusion}

Direct simulations of premixed flames in turbulent shear flow are used in this study to examine the effect of a mean shear motion on the flame surface dynamics. The shear is uniform in the unburnt gas, and simulations are performed for different values of the mean shear rate, $S$. A detailed analysis of the rate of change of flame surface densities is performed, based on the exact $\Sigma$-equation. In particular, the time evolution of the total flame stretch, $\widehat{\kappa}$, is compared for different values of $S$. In the expression for the total flame stretch, the production term by hydrodynamic straining is decomposed into two components: a component, $\widehat{A_{T}}$, that depends explicitly on the mean rate of deformation; and a component, $\widehat{a_{T}}$, that is determined by the turbulent flow field.

The data show that while $\widehat{\kappa}$ scales with the total shear, $S t$, it does not depend on the turbulence-to-mean shear time scale ratio, $S \tau_{0}$. This is a surprising result since the turbulence is strongly influenced by this parameter. This result implies that the principal effect of changing the mean shear rate, $S$, is to re-scale the time evolution of flame stretch. For instance, the results show that for $0 \leq S t \leq 10$, the rate of change of flame surface area is small; in contrast, for $S t>10$, a transition to a phase of rapid growth is observed. The main effect of $S$ is then to make that transition occur faster. Note also that, while flame stretch scales with $S t$, sirice the total flame surface area, $\left\langle S_{V}\right\rangle$, is related to the time integral of stretch, $\left\langle S_{V}\right\rangle$ is found to scale both with $S t$ and $S$.

The relative insensitivity of $\widehat{\kappa}$ to changes in $S \tau_{0}$ was further studied by examining the direct contribution of the applied shear to $\widehat{A_{T}}$. This contribution is always small in the simulations. It is shown to scale with the product of the magnitude of the mean velocity gradient and a coefficient that depends on the flame wrinkling. The magnitude of that coefficient is determined by competing effects: a positive turbulent effect and an initially positive and subsequently negative effect controlled by the mean rotation component of the applied shear. It is argued that this coefficient scales with the inverse of a characteristic turbulent time divided by $S$ so that at high shear rates, the increased mean velocity gradient is multiplied by a decreased coefficient and there is no net variation of $A_{T}$.

Finally, the question of which term in the $\Sigma$-equation is responsible for the transition to rapid growth of flame surface area, at $S t>10$, is studied by comparing $\widehat{a_{T}}$
and $\widehat{A_{T}}$. Results show that $\widehat{a_{T}}$ is increasingly dominant in the expression for flame stretch; the main effect of the applied shear is to increase the turbulent straining motions, and $\widehat{A_{T}}$ may be neglected in the models.

\section{REFERENCES}

Ashurst, W. T., Peters, N. \& Smooke, M. D. 1987 Numerical simulation of turbulent flame structure with non-unity Lewis number. Combust. Sci. Tech. 53, 339-375.

BoRGHI, R. 1990 Turbulent premixed combustion: further discussion on the scales of the fluctuations. Combust. Flame. 80, 304-312. 
Bray, K. N. C. 1990 Studies of the turbulent burning velocity. Proc. $R$. Soc. Lond. A. 431, 315-335.

CANDEl, S. M. \& PoInsot, T. 1990 Flame stretch and the balance equation for the flame surface area. Combust. Sci. Tech. 70, 1-15.

Candel, S. M., Veynante, D., Lacas, F., Maistret, E., Darabiha, N. \& Poinsot, T. 1990 Coherent flame model: applications and recent extensions. in Series on Advances in Mathematics for Applied Sciences. World Scientific.

CANT, R. S., Pope, S. B. \& Bray, K. N. C. 1990 Modelling of flamelet surfaceto-volume ratio in turbulent premixed combustion. Twenty-Third Symp. (International) on Combust. 809-815. The Combustion Institute.

Darabiha, N., Giovangigli, V., Thouvé, A., Candel, S. M. \& Esposito, E. 1987 Coherent flame description of turbulent premixed ducted flames. in Proc. of the France-USA Joint Workshop on Turbulent Combustion. Springer Verlag.

Duclos, J. M., Poinsot, T. \& Veynante, D. 1993 A comparison of flamelet models for premixed turbulent combustion. Combust. Flame. 95, 101-117.

HAworTh, D. C. \& PoINSOT, T. 1992 Numerical simulations of Lewis number effects in turbulent premixed flames. J. Fluid Mech. 244, 405-436.

Holt, S. E., Koseff, J. R. \& Ferziger, J. H. 1992 A numerical study of the evolution and structure of homogeneous stably stratified sheared turbulence. $J$. Fluid Mech. 237, 499-539.

LEE, M. J., KIM, J. \& MoIN, P. 1990 Structure of turbulence at high shear rate. J. Fluid Mech. 216, 561-583.

LELE, S. K. 1992 Compact finite difference schemes with spectral-like resolution. J. Comp. Phys. 103, 16-42.

LEONARD, M. J. \& HiLl, J. C. 1992 Mixing and chemical reaction in sheared and nonsheared homogeneous turbulence. Fluid Dynamics Res. 10, 273-297.

Maistret, E., Darabiha, N., Poinsot, T., Veynante, D., Lacas, F., CanDEL, S. M. \& EsPosito, E. 1989 Recent developments in the coherent flamelet description of turbulent combustion. in Proc. 9rd Int. SIAM Conf. on Numerical Combustion.

MANTEL, T. \& Borghi, R. 1991 A new model of premixed wrinkled flame propagation based on a scalar dissipation equation. in Proc. 19th ICDERS.

MARble, F. E. \& BroAdWELl, J. E. 1977 The coherent flame model for turbulent chemical reactions. Project Squid Technical Report. TRW-9-PU.

MAXEY, M. R. 1982 Distortion of turbulence in flows with parallel streamlines. $J$. Fluid Mech. 124, 261-282.

Meneveau, C. \& Poinsot, T. 1991 Stretching and quenching of flamelets in premixed turbulent combustion. Combust. Flame. 86, 311-332. 
Nomura, K. K. \& Elghobashi, S. E. 1992 Mixing characteristics of an inhomogeneous scalar in isotropic and homogeneous sheared turbulence. Phys. Fluids A. 4, 606-625.

Peters, N. 1986 Laminar flamelet concepts in turbulent combustion. Twenty-First Symp. (International) on Combust. 1231-1250. The Combustion Institute.

Poinsot, T., Veynante, D. \& CANDel, S. M. 1991 Quenching processes and premixed turbulent combustion diagrams. J. Fluid Mech. 228, 561-605.

PoINSOT, T. \& LELE, S.K. 1992 Boundary conditions for direct simulations of compressible viscous flows. J. Comp. Phys. 101, 104-129.

POPE, S. B. 1988 Evolution of surfaces in turbulence. International J. Engng. Sci. 26, 445-469.

PoPE, S. B. 1990 Computations of turbulent combustion: progress and challenges. Twenty-Third Symp. (International) on Combust. 591-612. The Combustion Institute.

Pope, S. B. \& Cheng, W. K. 1988 The stochastic flamelet model of turbulent premixed combustion. Twenty-Second Symp. (International) on Combust. 781789. The Combustion Institute.

Rogallo, R. S. 1981 Numerical experiments in homogeneous turbulence. NASA Technical Memorandum. 81315.

Rogers, M. M. \& MoIN, P. 1987 The structure of the vorticity field in homogeneous turbulent flows. J. Fluid Mech. 176, 33-66.

Rogers, M. M., Mansour, N. N. \& Reynolds, W. C. 1989 An algebraic model for the turbulent flux of a passive scalar. J. Fluid Mech. 203, 77-101.

Rohr, J. J., Itsweire, E. C., Helland, K. N. \& Van Atta, C. W. 1988 An investigation of the growth of turbulence in a uniform-mean-shear flow. J. Fluid Mech. 187, 1-33.

Rutland, C. J. \& Trouvé, A. 1993 Direct simulations of premixed turbulent flames with non-unity Lewis numbers. Combust. Flame. 94, 41-57.

TAvoularis, S. \& CoRRSIN, S. 1981 Experiments in nearly homogeneous turbulent shear flow with a uniform mean temperature gradient. Part 1. J. Fluid Mech. 104, 311-347.

Tavovlaris, S. \& KARNIK, U. 1989 Further experiments on the evolution of turbulent stresses and scales in uniformly sheared turbulence. J. Fluid Mech. 204, 457-478.

Townsend, A. A. 1976 The structure of turbulent shear flow. 2nd ed., Cambridge University Press.

TrouvÉ, A. 1993 The evolution equation for the flame surface density in turbulent premixed combustion. in CTR Annual Research Briefs - 1992, Center for Turbulence Research, Stanford Univ./NASA-Ames. 
TrouvÉ, A. \& PoInsot, T. 1993 The evolution equation for the flame surface density in turbulent premixed combustion. J. Fluid Mech. (submitted for publication).

Williams, F. A. 1985 Combustion theory. 2nd ed., Benjamin Cummings.

Wray, A. A. 1990 Minimal storage time-advancement schemes for spectral methods. J. Comp. Phys. (submitted for publication). 